Erschienen in: Stickel, Gerhard (Hrsg.): Deutsche Gegenwartssprache. Tendenzen und Perspektiven. - Berlin, New York: de Gruyter, 1990. S. 66-87. (Institut für deutsche Sprache. Jahrbuch 1989)

\title{
UWE PÖRKSEN
}

\section{"Unsere politische Sprache ist leer und bewegt fast nichts mehr." - Politik als Sprache und literarische Form}

In diesem Vortrag versuche ich, eine Frage zu stellen, weniger, auch schon eine Antwort zu geben. Die Frage gilt den Textgattungen des Politischen oder, wie wir auch sagen könnten, der Metapolitik. Ich vermute, daß die allgemeinere Einsicht in die Notwendigkeit, eine unabhängige, gegenüber der gefährlichen Liäson von Naturwissenschaft und Ökonomie selbständige Politik zu erkämpfen und demokratische Fermente in unserer Gesellschaft auszubreiten, davon abhängen wird, ob wir über wirksame Gattungen der Metapolitik verfügen und sie sich zu einer auf Anerkennung beruhenden fußlosen "Institution" politischer Kritik vereinigen. Ich gehe aus von einem historischen Beispiel.

I.

Im Frühjahr 1819 verließ der dreißigjährige Livländer Carl Gustav Jochmann seine Heimatstadt Riga, kaum anders als fünfzig Jahre vor ihm Herder, um in Deutschland oder jedenfalls in Westeuropa das Leben eines freien politischen Schriftstellers zu führen. Er war Jurist und hatte sich als Advokat in Riga ein Vermögen erarbeitet, von dem er leben konnte. Seine Freunde waren Bankiers, Politiker, Kaufleute, und der Schweizer Schriftsteller Zschokke. Er hielt sich zunächst in Deutschland auf und schrieb im Juni 1819 einen dreißig Seiten langen politischen Brief an den Freund Sengbusch in Riga.

Dieser Brief ist ein großartiges Dokument, das in mancher Hinsicht das Junge Deutschland antizipiert. Jochmann verrät schon zu Beginn, daß ihn die Frage der politischen Textgattungen beschäftigt. Er beginnt mit der Geschichtsschreibung, von der er die höchste Meinung hat, wirft einen Blick auf England, Italien, Frankreich, und urteilt: ,in Deutschland gibt es fast nichts, als Stammbäume und einen Haufen bedeutungsloser fürstlicher Familiengeschichten, in die des Volkes Geschichte zusammengeschrumpft ist." Historie sei hier "das Kammermensch" jedes kleinen Dynasten. (Jochmann 1836, S. 4). ${ }^{1}$

Als zweites spricht er von jenen 'Denkwürdigkeiten', 'Memorabilien', wie

1 Vgl. auch den Wiederabdruck des Briefes bei Werner Kraft 1967, S. 33-41; Zitate: S. 33, 34. Schon ein Blick auf die Geschichtsschreibung Rottecks legt die Frage nahe, ob dieses Urteil Jochmanns gerecht ist. 
sie speziell in Frankreich verborgen aufgezeichnet worden sind und erst postum ans Tageslicht gelangten. Er führt sie also als eine ursprünglich oppositionelle Gattung ein und gibt dann eine genauere Analyse:

Denkwürdigkeiten freilich verhalten sich zur Geschichte nur, wie das Ankleidezimmer zur Bühne, die Küche zum Speisesaale. Was wir haben, zeigen diese, wie wir dazu kommen, jene, und mit beiden mögen sie auch das gemein haben, daß sie nur zu oft die Lust an den Schauspielern und an den Gerichten verderben.

Denkwürdigkeiten erlauben also einen Blick hinter die Kulissen oder in den Kochtopf, und daß einem dabei übel werden kann, läßt sich z.B. aus Straßenstudien zur Zeit der Französischen Revolution entnehmen, der 'Physiognomie von Paris am 10. August 1792 und am 21. Jänner 1793', die Jochmann auf Grund von Gesprächen mit dem in Paris lebenden preußischen Grafen Gustav von Schlabrendorf überliefert hat (Jochmann 1836, S. $160 \mathrm{ff}$.). Auf die Wahrheit komme es an, meint er, und erkennt den Memorabilien drei Vorzüge zu: sie seien 1. unterhaltender als die oft gravitätischen historischen Werke, 2. planlos, vielseitiger und umfänglicher im Erfassen der Zeitgeschichte, 3. die Persönlichkeit des Erzählers komme in ihnen stärker zum Ausdruck, was aber nur ein Vorzug sei, wenn die Autoren ihre eigenen Ansichten auch vorzutragen wagten.

Diese Zivilcourage vermißt er in Deutschland. Die politische Luft im Juni 1819, drei Monate nach der Ermordung Kotzebues durch den Theologiestudenten Carl Ludwig Sand, sei schwül und beengend. Der Kleinmut der Schriftsteller und eine gröbere oder feinere Zensur entziehe die interessantesten Dinge der Öffentlichkeit, man erfahre sie nur im vertrauten Gespräch.

Damit sie Ihnen nicht ganz entzogen werden, habe ich mir vorgenommen, Ihnen in meinen Briefen eine Art Zeitung im Manuscripte zu liefern, die, ohne allen Plan, alles enthalten soll, was an interessanten Notizen durch eigene Erfahrung oder aus glaubwürdigen Quellen zu meiner Kenntnis gelangt ist. (Jochmann 1836, S. 6).

Jochmann verspricht eine Art Zeitung als Samisdat; auch hier gibt er eine nähere Gattungsbestimmung: „ohne allen Plan.” Dieser politische Brief hat die Form eines lockeren, scheinbar planlosen Essays, der für das Deutschland der Karlsbader Beschlüsse ein breit angelegtes Öffentlichkeitsgemälde entwirft.

Das Gemälde ist ungemein lebendig und vielseitig; fragt man sich, wie dieser Eindruck erzeugt wird, so fallt auf, daß Jochmann von Gattung zu Gattung springt - sei es, daß er über die eine spricht oder die andere erprobt. 
- das viel diskutierte politisch brisante Buch 'Welt und Zeit' und nennt seinen mutmaßlichen Verfasser (es handelt sich um eine Sammlung moralisch-politischer Aphorismen eines Frankfurter Juristen und Geschäftsmannes), er gibt eine

- Reportage der offiziellen Reaktion und der Stimmung des Volkes nach der Ermordung Kotzebues (Mannheim, wo der Mörder inhaftiert ist, ist ein Ziel aller Ferienreisen), fügt

- eine kurze bissige Portraitskizze Kotzebues ein und schließt dann, "einem Brauche der Zeit gemäß, der bedeutsam genug allen Unterhaltungsstoff in politischen und nichtpolitischen teilt", ein

- "nichtpolitisches Feuilleton" an. Hier

- bespricht er Bücher (z.B. Goethes 'Kunst und Altertum', das ihm wie das Werk eines pensionierten Hofmarschalles erscheint),

- gibt eine Tendenzmeldung (in Deutschland grassiere ein neuer Mystizismus, eine Art Schirokko der geistigen Welt),

- berichtet von einer wissenschaftlichen Revolution, die der Medizin bevorstehe (die Kurmethode des Dr. Hahnemann in Leipzig, die Homöopathie). Den Schluß bildet

- ein Novellenstoff, der kleistschen Zuschnitt hat, und etwas

- Klatsch.

In dem Augenblick, wo der junge Advokat Jochmann aus Osteuropa aufbricht, um sich im Westen zum politischen Schriftsteller zu mausern, reflektiert er über die Gattungen politischer Rede und nennt und erprobt in seinem politischen Brief nach Riga auch gleich eine Anzahl von ihnen.

Gattungen sind Ordnungsformen, die einen Gegenstand nach mehr oder weniger festen Spielregeln erschließen, Okulare, Perspektiven, die Aspekte eines Realitätsausschnitts erkennbar werden lassen, indem sie ihn durch die Regeln der Gattung filtern. Sie setzen einen erprobten Rahmen - erprobt meist in sozial vorgeprägten und wiederkehrenden Situationen -, innerhalb dessen ein Weltausschnitt erkannt, erwogen und befunden, geordnet wird. Sie stimulieren und lenken den Zugriff. Ihre Regeln werden meistens so wenig bewußt angewendet wie die der Grammatik; ihre Geltung wird am spürbarsten, wenn sie verletzt werden. Gerade dann zeigt sich auch, daß an die jeweilige Gattung eine bestimmte Konvention der Darstellung bis hin zur charakteristischen Wortwahl gebunden ist.

Es gibt eine Kunstform des Essays - auch Goethe verwendet sie in seiner Stellungnahme zu dem Pariser Akademiestreit, den 'Principes de Philosophie Zoologique' von $1830 / 32$-, die in einer lockeren Reihe verschie- 
dene Gattungen verbindet und durch sie, als durch ebenso viele geregelte Perspektiven auf verschiedene Realitätsausschnitte, einen Gegenstandsbereich vielseitig anleuchtet. Das geschieht in dem hier besprochenen Öffentlichkeitsgemälde.

Jochmann hat ein entschiedenes Gattungsbewußtsein, einen ausgeprägten Sinn für Form; seine weitere schriftstellerische Tätigkeit wird darin bestehen, in einer beachtlichen Anzahl von Gattungen politisch und moralisch Stellung zu nehmen und Muster politischer Reflexion vorzulegen. Dabei bedient er sich zum Teil solcher Formen, für die er wohl eher in Frankreich und England eine Tradition vorgefunden hat; ich verstehe seine Absicht so, daß er versucht hat, Formen politischer Reflexion in Deutschland heimisch zu machen, sie zu implantieren, um durch sie an der Schaffung einer politischen Öffentlichkeit, eines politischen Bürgertums mitzuwirken. Nicht so sehr die Rhetorik als Stillehre - sie war diesem baltischen Juristen noch selbstverständlich - sondern Gattungen der Metapolitik wollte er übertragen, so scheint es.

Speziell durch diese Gattungen entwirft er sich als den Typus eines politischen Schriftstellers, der bei uns nie recht heimisch geworden ist.

Er schrieb die

- wissenschaftlich fundierte Streitschrift, die einen umfangreichen Sektor wider den Strich bürstet (eine historische Kritik des Adels und des Protestantismus, eine Sprachkritik);

- politischen Essays, die für einen neuen Begriff den Blick öffnen wollen (z.B. für eine neue Deutung Robespierres),

- Glossen, die meist einen Satz, einen Gedanken in einem gedrängten Kurzessay durchführen,

- Aphorismen, die ihre Entstehung meist dem Widerspruch verdanken,

- Reisebilder,

- Denkwürdigkeiten,

- Anekdoten,

- Portraits, die er Charakter-Umrisse nannte.

Jürgen Schiewe hat in seinem gerade erschienenen Buch 'Sprache und Öffentlichkeit. Carl Gustav Jochmann und die politische Sprachkritik der Spätaufklärung', das die historischen Konturen dieses halb verschollenen Autors erkennbar macht und, wie ich glaube, geeignet ist, die in ihm ruhenden Energien zu entbinden, Schiewe hat dargestellt, auf welche sprachgeschichtliche Situation Jochmann reagiert: nämlich auf ein seit dem 18. Jahrhundert auf der Sprache lastendes Übergewicht der Schrift, ihr Defizit an Mündlichkeit, und er hat vorgeführt, daß Jochmann den 
politischen Gattungen ein mündliches Element bewußt einverleibt hat (Schiewe 1989). ${ }^{2}$

Man kann schon in der Rhetorik die Kunst sehen, einem geschriebenen Text die Physiognomie der mündlichen Redesituation einzuschreiben. Die Rhetorik simuliert Mündlichkeit. Aber noch ein anderer Vorzug der mündlichen Sprachluft scheint Jochmann zu beschäftigen. Er beobachtet in dem Buch 'Über die Sprache' (1828), daß in einer mündlichen Gesellschaft der Vers die Aufgabe erfüllt, die in der schriftlichen vom Buchstaben übernommen wird. Die Form stützt das Gedächtnis, sie sorgt für die Aufbewahrung eines Gedankens im Ohre (Jochmann 1968, S. 255 ff., 282).

Wollte Jochmann durch seine rhetorisch artikulierten politischen Gattungen einer von der Schrift bestimmten Kultur den Vorzug der Formen, Prägungen für das Gedächtnis zu sein, erhalten, sie hinüberretten? Seine Formen, diese Reisebilder und Denkwürdigkeiten, Charakter-Umrisse und Erfahrungsfrüchte, ermöglichen eine Art Gestaltwahrnehmung. Das Artikulierte haftet. Erst die Prägung durch eine Form ermöglicht einen Diskurs, der den Tag überdauert, weil die Politica dem öffentlichen Gedächtnis zur Verfügung stehen.

$\mathrm{Zu}$ der Haltung, in der Jochmann schreibt, gehört ein Vergnügen an der Form: an enigmatischer Kürze und der logischen Verschlüsselung, dem Paradox und dem randscharfen Bild. Es gibt hier ein pures Vergnügen, eine reine Lust.

Die Form scheint aber noch eine weitere Bedeutung zu haben: sie entlastet. Sie verleiht der Gereiztheit des moralischen Nervs Ausdruck und macht den inneren Aufruhr weltmöglich. Sie überführt, was an kritischem Potential in diesem reizbaren Autor gärt, durch die klärende Form in die öffentliche Diskussion.

Die Form ist schließlich eine Waffe, sie spitzt den Gedanken zu und versieht ihn mit dem richtigen Gefieder, so daß er trifft und sitzt. Die erwähnten Formen dienen neben der Erinnerung und Klärung auch dem Angriff.

Jochmanns Sprache und literarische Form vermitteln also ein konturiertes Bild:

- Form stützt das öffentliche Gedächtnis.

- Sie erhält der Schrift einen Schein von Mündlichkeit.

2 Siehe besonders die Kapitel VI,3 und VII,2. 
- Sie ist als Gattung eine Ordnungsform, Spielregel des Zugangs zur Realität, Instrument der Erkenntnis und Urteilsfindung.

- Sie ist ein intellektuelles Vergnügen und daher gesellig.

- Sie macht Empörung weltmöglich und diskutabel.

- Sie ist eine scharfe, zugespitzte Waffe.

II.

Wir stoßen also im frühen 19. Jahrhundert auf Gattungen des Politischen, die einerseits oberhalb der Ebene der Tagesaktualität und der Zeitungen angesiedelt sind und andererseits nicht aus der wissenschaftlichen Werkstatt hervorgehen, nicht deren Anspruch auf Exaktheit und Objektivität unterworfen sind. Jochmanns politischer Brief und seine späteren Arbeiten sind Musterstücke aus diesem Zwischenbereich: aus dem Bezirk eines öffentlichen historischen und politischen Gedächtnisses, der grundsätzlicheren Einordnung des Tagesgeschehens, der politischen Moral und Klugheitslehre. Mit einem Wort: der praktischen Philosophie.

Von diesem Beispiel ausgehend, möchte ich meine Frage stellen: Wo sind heute die Gattungen der Metapolitik anzutreffen? Welches sind gegenwärtig die politischen Ausdrucksmittel mit öffentlicher Langzeitwirkung? In welchen Textgattungen findet die über den Tag hinausweisende politische und moralische Reflexion statt?

Einige der von Jochmann erprobten Formen, insbesondere die Denkwürdigkeiten, Anekdoten und Charakter-Umrisse, sind verschwunden. Für die Glossen, wie er sie verstand, diese Kurzessays, sind vielleicht Adornos 'Minima Moralia' ein spätes Beispiel: Für seine politischen Aphorismen, diese sog. 'Stilübungen' oder 'Erfahrungsfrüchte', wüßte ich keins.

Nun ist es nicht das Ziel, vorzuschlagen, man möge in der Gegenwart 'Denkwürdigkeiten' und 'Charakterskizzen' schreiben, selbstverständlich nicht. Jochmann wäre überdies ein schlechtes Beispiel, wenn man mit der goldenen Elle einer verklärt gesehenen Vergangenheit der Gegenwart beikommen wollte. Er war ein Außenseiter, lebte als russischer Untertan bis zu seinem frühen Tod 1830 meist im Badischen, veröffentlichte zu seinen Lebzeiten nur weniges anonym. Erst sein Nachlaß, den Zschokke 1836-38 herausgab, erregte für kurze Zeit Aufsehen.

Jochmann hing also in der Luft. Ich gehe von ihm aus als von einer konturierten Folie: wenn die abstrakte Leistung seiner politischen Formübungen richtig beschrieben ist - wo findet sich gegenwärtig ihr Äquivalent? Welches ist zur Zeit - mutatis mutandis - der geeignete Rahmen für Metapolitik? 
Fast hundert Jahre nach ihm verfügt noch einmal Karl Kraus, für den Sprache und Form in gleich hohem Grade Ausdruck der politischen Moral sind, über ein großes Repertoire von Gattungen. Er schrieb nicht nur Essays, Glossen und Aphorismen, also Prosa, sondern auch Poesie, Gedichte, Epigramme und Parodien, Satiren und Schauspiele. Auch er ein Außenseiter und Privatunternehmer, jedoch nicht ohne öffentliche Wirkung. Mancher fragt sich allerdings, ob er politisch etwas ausgerichtet hat.

Noch einmal: Welche Gattungen der Metapolitik haben die einstige praktische Philosophie beerbt und sind zur Zeit in Gebrauch?

Es gibt keine Geschichte dieser Gattungen; in der Literaturwissenschaft findet man zahlreiche und erhellende Studien zu einzelnen Genres wie Aphorismus und Essay, aber die deutsche politische Aphoristik und Essayistik, die es durchaus gibt, erscheint nur als Randthema (Rohner 1966 und neuerdings Schote 1988).

Ergiebig, ja, allzu ergiebig wäre es, wenn wir die Geschichte der politischen Rede in Deutschland berücksichtigen wollten und der Frage nachgehen würden, ob der Topos von einem im 18. Jahrhundert beginnenden Verfall der rhetorischen Tradition in Deutschland und der hier vorherrschenden Verachtung der Beredsamkeit berechtigt ist. Wir müssen dies Thema hier ausklammern. Es würde diesen Versuch sprengen. Die Rhetorik versteht sich aber - auch theoretisch - als 'ars oratoria', als Lehre von der Anordnung und den Stilelementen der überzeugenden Rede, nicht als Lehre von den Textgattungen - und sie gibt uns über Textgattungen $\mathbf{m}$. W. keine Auskunft.

Auch ein Blick in die Soziologie führt vorläufig nicht weiter. In Jürgen Habermas 'Strukturwandel der Öffentlichkeit' (1962) sind die Gattungen des öffentlichen politischen Räsonnements kein Thema. Richard Sennetts 'Verfall und Ende des öffentlichen Lebens' (1974, deutsch 1983) läßt uns in dieser Hinsicht nur nicht im Stich, wenn wir dem 'Image' nachfragen. Hier ist die Literatur umfangreich (Habermas 1974 und Sennett 1983).

Nachdem ich wenig fand, habe ich mich bei den Politologen umgehört, und es scheint sich zu bestätigen. Es gibt keine Geschichte der politischen Gattungen und ihrer Übergangsformen zur Metapolitik. Dabei wäre eine solche Geschichte höchst aufschlußreich. Das Verschwinden alter und die Erfindung neuer Gattungen wäre ein Indikator des Öffentlichkeitswandels. Wer sie schreiben wollte, müBte allerdings wohl gleichzeitig Politologie und Soziologie, Literaturgeschichte und Mediengeschichte, Rhetorik und Textlinguistik im Auge haben. 
Im Vergleich zu dem bisher Gesagten läßt sich ein klares Bild von den heute in Gebrauch befindlichen journalistischen Textgattungen gewinnen. Man stöBt hier, vom Wörterbuch zur Publizistik, über Einführungen in den praktischen Journalismus bis zu mehr oder weniger wissenschaftlichen Analysen auf eine umfangreiche Literatur,${ }^{3}$ in der sich zumindest schriftlich - ein ausgeprägtes BewuBtsein von Gattungen und Gattungsregeln zu erkennen gibt. Uns interessieren hier weniger die informierenden Gattungen wie Nachricht, Bericht, Dokumentation, als die interpretierenden und kommentierenden, die werten: Magazinbeitrag, Diskussion, Portrait, Brief, Feuilleton, Essay, Kommentar, Leitartikel, Glosse, Kolumne. Überlegen wir aber, was wir intuitiv von diesen $\mathrm{Ge}$ brauchsformen wissen, und schauen wir uns ihre Umschreibungen an, so erscheinen sie kaum als die Beispiele öffentlichkeitswirksamer politischer Klugheitslehre, die wir suchen. Oder doch?

Wie steht, zwischen Tagespresse und Wissenschaft, der Bereich politischer Moral da? Ist er besetzt, und durch welche Gattungen? Kommen hier wissenschaftliche Gattungen in betracht, oder ist vielleicht die Wissenschaft gerade nicht in der Lage, sie ernst zu nehmen? Entzieht sie ihnen ihr Gewicht, sorgt sie durch ihre Ansprüche an exakte Empirie und Objektivität für eine Ausdünnung der politischen Diskussion?

Ist die Verbindung von Poesie und Politik die Gattung, die hier gesucht wird? Um es gleich zu sagen: sie ist es nicht. Die Tendenzliteratur, die unsere Republik von Anfang an begleitet hat, und die nach 1968 vorübergehend zum dominierenden Typus wurde, ist ein viel zu umfängliches und vielschichtiges Phänomen, als daß ich nebenbei auf sie eingehen könnte. ${ }^{4}$ Aber sie liegt, wie die Rhetorik, auch nicht ganz auf dem hier eingeschlagenen Weg.

Einen erhellenden und weitreichenden Hinweis erhalte ich durch den Freiburger Politikwissenschaftler Wilhelm Hennis, durch sein Buch 'Politik und praktische Philosophie. Eine Studie zur Rekonstruktion der politischen Wissenschaft' (Hennis 1963). Hennis These lautet, die politische Wissenschaft sei keine 'junge', sondern eine uralte, auf Aristoteles zurückgehende Wissenschaft. Er nennt sie 'praktische Philosophie'. Diese antike Disziplin sei "unter der Herrschaft des modernen Wissenschaftsbegriffs so gut wie vollständig zum Erliegen gekommen" (S. 106). Der

3 Vgl. hierzu Dovifat/Wilke 1976; Noelle-Neumann/Schulz 1971; Koszyk/Pruys 1970; La Roche 1987; Roloff 1982; Belke 1973; Burger 1984; Camen 1984; Bentele/Ruoff 1982; Lüger 1983; Straßner 1975; Dieckmann 1981.

4 Das Thema möchte ich an anderer Stelle aufgreifen. 
Universalitätsanspruch naturwissenschaftlicher Denkformen hat ihm zufolge ein Fach, an das an sich ganz andere Ansprüche zu stellen sind als die der permanenten Aufdeckung neuer gesetzmäfiger Zusammenhänge und ihrer zweifelsfreien Herleitung aus Wirkungsursachen (S. 96, 124), in Mißkredit gebracht und dazu geführt,

daß für die Politik kein eigener Gegenstand übrigblieb. Politik kann nun nicht mehr verstanden werden als vernünftige Ordnung menschlichen Zusammenlebens, sondern sie ist Folge, Funktion, Reflex irgendwelcher als solcher unpolitischer Kausalitäten (S. 124).

An sich hatte die praktische Philosophie ihre eigene Findekunst, ihre eigene Methode gehabt, eine Technik, die Gesichtspunkte zu finden und die Argumente zu durchlaufen und zu wägen - sie lag in der Dialektik und Rhetorik bereit: Hennis beschreibt sie in dem Kapitel VI 'Topik und Politik' und demonstriert ihre Auflösung durch Bacon, Hobbes, Descartes. Das ist eine grundsätzlichere Beobachtung als die häufig zu hörende Klage über den Verfall der Rhetorik; der philosophische Rahmen, in dem Rhetorik und Dialektik ihren Ort hatten, ist gefallen. Die Folge:

Das praktische Denken ist orientierungslos geworden; ... Was hülfe es, wenn der Mensch die ganze Wahrheit der Natur erkannt, aber die des tätigen Lebens darüber vergessen hätte, nur weil sich ihm diese nicht clare et distincte preisgibt? (S. 107).

Die Aktualität solcher Sätze hat sich in den letzten 25 Jahren erhöht. Es ist ja schon für Hennis nicht nur die politische Wissenschaft, die ihren Gegenstand verloren hat, sondern die Politik selbst. Diese Enteignung der Politik entspricht inzwischen der alltäglichen Erfahrung. Uns erreicht ein Bandwurm von Hiobsbotschaften, Nachrichten, die in den Alpträumen unsrer Kindheit noch nicht vorgesehen waren. Aber nichts Einschneidendes geschieht. Politik findet kaum statt.

Ulrich Beck nennt in seiner nicht genug zu bewundernden Analyse, 'Risikogesellschaft', das, was stattfindet, geradezu eine 'Nichtpolitik'. Die bestimmenden Entscheidungen fallen in Positionen, die ohne politische Verantwortung handeln:

- in den Labors der Forschung.

- in den Chefetagen der Wirtschaft.

Die Situation droht ins Groteske umzuschlagen: Die Nichtpolitik beginnt, die Führungsrolle der Politik zu übernehmen. Die Politik wird zur öffent lich finanzierten Werbeagentur für die Sonnenseiten einer Entwicklung, die sie nicht kennt und die ihrer aktiven Gestaltung entzogen ist.

Die Reichweite der Gesellschaftsveränderungen verhält sich umgekehrt 
proportional zu deren Legitimation, ohne daß dies an der Durchsetzungsmacht des zum 'Fortschritt' verklärten technischen Wandels etwas ändern würde (Beck 1986, S. 358), 329).

Die Sprache der Politik dokumentiert nichts anderes: sie ist über weite Strecken nur schwer von der Sprache einer durch die Industrie finanzierten Werbeagentur zu unterscheiden.

Es sei mir erlaubt, als dritten Gewährsmann einen ungewöhnlichen Politiker anzuführen: „Unsere politische Sprache ist leer und bewegt fast nichts mehr:" Der Satz ist von Erhard Eppler, ich entnehme ihn mit seiner freundlichen Erlaubnis einem Brief, den er mir vor zwei Jahren (25.2.1987) zu einem Vortrag über 'Die lautlose Gewalt der Amöbenwörter' geschrieben hat. ${ }^{5}$

Der Satz hat mich beschäftigt. Trifft er zu? Wählen wir den Satz eines normalen Politikers als Beispiel: „Zukunft ist wichtiger als Freizeit." Der Satz erweckt den Eindruck großräumiger Leere - er umfaßt die Bundesrepublik oder notfalls die ganze Welt und ihre Zukunft. Aber: bewegt er nichts? Es ist kein Nullsatz, so wenig wie das Medium, durch das man ihn am schönsten verbreiten könnte, ein Nullmedium ${ }^{6}$ ist: er hält durchaus etwas in Bewegung. Aber es ist kein politischer Satz, sondern ein Satz im Sinn der durch die Allianz von Naturwissenschaft und Ökonomie durchgesetzten Zukunftsdefinition. Die von ihm in Gang gehaltene Bewegung ist gerade nicht Politik. Die Politik reflektiert die in der Öffentlichkeit herumgeisternden Idole, die von der genannten Allianz aufgerichtet sind, spiegelt, moduliert diese Wunschbilder und - läßt den Karren laufen.

So verstehe ich den Satz: „Unsere politische Sprache ist leer und bewegt fast nichts mehr." Es fehlt die Selbständigkeit des Politischen, ja, selbst ein öffentliches Verlangen nach solcher Selbständigkeit. Es fehlt eine selbständige politische Sprache.

5 Erhard Eppler war durch eine Zeitungsmeldung (ap), die unter der Überschrift 'Sprache einer leisen Diktatur' in der FR erschien und mir eine Verlangsamung meiner Post eintrug, auf den Vortrag aufmerksam geworden und hatte ihn erbeten. Der Vortrag stand in kurzer Fassung am 17.1.1987 in der Frankfurter Allgemeinen Zeitung; er entspricht ziemlich genau dem fünften Kapitel meines Buches 'Plastikwörter. Die Sprache einer internationalen Diktatur. Stuttgart 1988'. Dazu Eppler: „Ihr Buch habe ich bekommen, aber noch nicht ganz gelesen, da ich noch dabei bin, einen heroischen, nicht völlig aussichtslosen Kampf gegen Plastiksprache im Grundsatzprogramm der SPD zu führen" (23.1.1989).

6 Von einem 'Nullmedium' spricht Hans Magnus Enzensberger (Enzensberger 1988). 
Wächst den genuin politischen Textgattungen unter solchen Umständen die Aufgabe zu, der Politik ihr Terrain zurückzuerobern? Lassen sich Beispiele solcher Sprache und Form finden?

Daß unser Bundeskanzler einen 'Brief an alle Deutschen' schreiben wird, können wir uns nicht leicht vorstellen. - François Mitterand hat im April 1988 einen dreizehn Zeitungsseiten langen 'Brief an alle Franzosen' geschrieben, in dem er sich zur Wiederwahl empfahl. Der Brief erinnere ihn stellenweise an den romantischen Dichter Lamartine, bemerkte einer der Gegenkandidaten Mitterands; er war nach allgemeinem Urteil sehr gut geschrieben. Mitterand berichtet darin von dem Besuch in der Ambulanz eines Pariser Hilfswerks:

Die kleine Volksansammlung, die sich darin drängte, war mit jener unendlichen Geduld gewappnet, welche den Entbehrlichen und längst Abgeschriebenen eigen ist; sie warteten, daß die Schlange vor dem Notfalldienst schwände. Und auch die freiwilligen Ärzte, Krankenschwestern, Sozialarbeiterinnen und Studenten waren unendlich geduldig; sie wußten, daß sich das Pensum menschlichen Unglücks niemals abarbeiten läßt und dennoch jeder mitmenschliche Akt Heil bringt. Einem jungen Mädchen liefen Tränen über die Wangen - sie schaute zur Decke und schwieg. Alle trugen das Kleid der Armut.

Wer kann eine so noble Prosa im Angesicht der Armut ohne Tränen hören oder lesen? Die Tradition der politischen Rhetorik und der Politik als literarischer Form scheint in Frankreich wenig gebrochen zu sein. (Roger de Weck 1988).

Nach dem Tod Edgar Faures stand in der 'Badischen Zeitung' ein Nachruf auf diesen 'Staatsmann und Grandseigneur' von der Hand des Frankreichkorrespondenten Fritz-Vannahme, der nicht nur gut geschrieben war, sondern auch typische Züge der älteren Portraitskizze hatte, bis hin zu dem von Faure in Umlauf gesetzten Bonmot: „Immer recht zu behalten, ist ein großer Fehler" (Badische Zeitung 31.3./1.4.1988). - Vermutlich war der Nekrolog französischen Zeitungen entnommen; man wurde an Jochmanns Charakter-Umrisse erinnert, diese lapidaren, lebendigen Standbilder, die von herausragenden Zeitgenossen einen scharf umrissenen Begriff vermitteln, von ihren Tugenden und Untugenden, und dadurch der politischen Konversation zu Verfügung stehen.

Es wäre interessant, die Kunst des politischen Portraits in der Bundesrepublik zu untersuchen. - Wir haben in einem Seminar Jochmanns Charaktere mit einem Portrait von Bundeskanzler Helmut Kohl verglichen, das Rolf Zundel am 23.1.1987, vor der letzten Bundestagswahl, ganzseitig in der 'Zeit' publiziert hat, unter der Überschrift ' „Man muß doch nur ja 
sagen" - Nach vielen Pannen und Schlappen: Helmut Kohl im Zenit des Selbstbewußtseins'. Das erste Resultat: der Vergleich ist kaum möglich. Im Fall Jochmann knappe Portraits, die noch auf der Vorstellung von typischen Charaktereigenschaften und Idealnormen, von Tugenden und Lastern beruhen, Arbeiten zudem eines unabhängigen Schriftstellers, auf der anderen Seite ein breit angelegtes Stimmungsbild, wo das Erscheinungsbild des Politikers eigentümlich gemischt ist aus Zutaten von der eigenen Person her und ihrer Profilierung durch ein öfentliches Bezugsnetz. Das Profil der Person scheint moduliert durch ein demoskopisch ermittelbares Wunschbild, Wahrheiten aus ihrem Mund erscheinen als Funktion erwartbarer Mehrheitsverhältnisse. Zundel befolgt die alte Regel der Poetik, bei der Darstellung der Person nur eine Eigenschaft hervorzuheben: es ist hier die massive Ruhe und Stetigkeit des Kanzlers. Das Festhalten an Personen z.B., und die damit verbundene Haltung des Bejahens.

Der gleiche Eindruck entsteht in einem zweiten, vor kurzem von der 'Zeit' (6.1.1989) publizierten Portrait: ' Ein Kanzler wie ein Eichenschrank." ... Viele stoßen sich an ihm, doch keiner kann ihn verrücken.' - Das Bild von 1987 ist freilich noch sehr unbestimmt, wenig informativ, zweideutig -; das Seminar vermochte sich nicht zu entscheiden, ob es positiv oder negativ gemeint war. Der Autor vollführt einen Balanceakt, zwischen der linken und der rechten Leserschaft, so scheint es, vielleicht auch zwischen den Besitzern und den Herausgebern des Blattes. Wir haben also die 'Charakterskizze' auf der einen Seite, auf der anderen das 'Image'. Die Geschichte des Portraits ist vermutlich ein besonders geeigneter Schlüssel zum Strukturwandel der Öffentlichkeit. Richard Sennetts Buch 'Verfall und Ende des öffentlichen Lebens. Die Tyrannei der Intimität' (Sennett 1983) unterstreicht diesen Eindruck oder z.B. ein Aufsatz Ortheils über den 'Staatsschauspieler' im MERKUR (Ortheil 1986). Man wünscht sich an Stelle eines solchen mit schwebender Unentschiedenheit geschriebenen Aufblasens von Stars das informierende, analytische, urteilende Portrait, - eine distanzierte und dezidierte, unabhängige Schreibweise.

Unser Thema wird heikel, wenn Nazivergangenheit und Terrorismus zur Diskussion stehen. Die Reflexion erhebt sich dann oft nur wenige Meter über einen Hexensabbath.

Die herausragende politische Ausnahme bildet bekanntlich auf beiden Feldern, auch dem des Terrorismus, der Bundespräsident. Die Ansprache vor dem Bundestag am 8. Mai 1985 zum Gedenken an das Ende des Zweiten Weltkrieges war eine befreiende Handlung: eine nüchterne und offene, klare, nur der Wahrheit verpflichtete Sprache. 
Sehr bedenkenswert in unserem Zusammenhang ist allerdings auch die Rede Philipp Jenningers, die, gehalten am 10. November 1988 zum Gedenken an die Progromnacht vor 50 Jahren, zum Eklat und zu seinem Sturz als Bundestagspräsident führte. Die Verletzung der Erwartungen, die durch die Situation gesetzt waren, war, in textlinguistischen Termini, ein Verstoß gegen die Regeln der Gattung, und so ist sie auch von vielen beschrieben worden - die falsche Rede zur falschen Stunde am falschen Ort ('Die Zeit', 18.10.1988) - aber selten ist die fundamentale Dimension dieser unsrer Regeln so klar erkennbar geworden. - Dabei ist die Rede, unabhängig von der Situation, in der sie gehalten wurde, ein nachdenklicher machendes Dokument als manche Gedenkrede, ein fast singuläres Beispiel öffentlicher politischer Anamnese, aus dem man, wie einem die Älteren sagen, ganz richtig erfährt, wie 1938 gedacht worden ist. Die 'Rollenprosa', von der Walter Jens sprach, ${ }^{7}$ hätte in anderem Zusammenhang ihren Sinn.

' „Es ist ein Volk ohne Geschichte!” sagen sie hohnlächelnd von den Amerikanern. Ein Volk ohne Geschichte ist ein Kind, ohne Sünden. Ein Volk ohne Geschichte ist ein Volk ohne Schandflecke.' - ein Aphorismus Jochmanns aus dem Jahr 1828 (Jochmann 1983, S. 146). ${ }^{8}$ Der Streit um die Frage, in welchem Maß die deutsche Geschichte im 20. Jahrhundert einzigartig ist, einzigartig in dem von Jochmann benannten Punkt, berührt den Nerv unsrer historischen Standortbestimmung - das klärungsbedürftigste Problem aus dem Bereich von Politik und Moral. Der sog. 'Historikerstreit' (Historikerstreit 1987) füllt einen Band. Es gab vergleichbare, wenn auch nicht ganz so tiefreichende Streitfragen in jüngster Zeit,

- den Streit um die Ursachen des Terrorismus, ausgelöst durch den Vorwurf an die Adresse der bundesrepublikanischen Schriftsteller, sie hätten diesen Terrorismus zu verantworten, und dokumentiert in den 'Briefen zur Verteidigung der Republik' (Duwe 1977),

- den Streit um die Bewertung des politischen Schriftstellers Heinrich Mann (vgl. Literaturmagazin 21, 1988), ausgelöst durch einen Aufsatz Reich-Ranickis in der Frankfurter Allgemeinen (15.8.1987).

Es handelt sich jeweils um geradezu musterhafte, komplexe, klärungsbedürftige Streitfälle. - Fallbeispiele, Polemik und Kontroversen auf dem Gebiet politisch-moralischer Reflexionen sind das, was wir suchen. Liest

7 In der gleichen Nummer der 'Zeit' vom 18.10.1988, die auch die Rede abdruckt.

${ }^{8}$ = Erfahrungsfrüchte II, Nr. 32. 
man die Dokumente, so stellt sich - von wenigen herausragenden Ausnahmen abgesehen - der Eindruck ein, wie sehr dieses Gebiet verwaist ist. Die Diagnose von Hennis trifft zu: es fehlt an praktischer Philosophie, an Philosophie und Techne für die Praxis. Die Kunst, Gesichtspunkte zu sammeln und Argumente zu durchlaufen, Schlüsse zu ziehen, die Kunst des Findens und der Disputation, diese Technik ist wenig ausgebildet. Die Debatte begibt sich ins Gestrüpp von Verdächtigungen und Unterstellungen, purem sprachlichen Ausdrucksverhalten, Denunziationen und Diffamierungen, und die Chance einer entscheidenden, klärenden, traditionsbildenden Sprache wird vertan.

Dieser Hexensabbath ist umso unerquicklicher, als er an die Vergangenheit fesselt, nicht von ihr befreit, so daß es an Kräften fehlt, sich der Gegenwart zu stellen. Mir jedenfalls erscheint das zweite als dringend notwendig.

IV.

Man könnte, versteht sich, über eine große Zahl politischer Gattungen sprechen, deren Sprache über den Tag hinaus wirken soll: Theodor Eschenburgs Aufsätze über Verfassungsrecht, das Grundsatzprogramm einer Partei oder das programmatische Buch eines Politikers, die Politikberatung - Egon Bahr berichtet in einem Gespräch mit Hans Magnus Enzensberger, wie aus einem Planungsstab der sechziger Jahre in langen Prozeduren die Formel 'Wandel durch Annäherung' hervorging (Bahr Sept. 1984) -; auch die kleinste Glosse kann so wirken, sie kann schlagartig eine Verletzung politischer Grundregeln ins Licht rücken. Ein Freiburger Beispiel:

Die Stadt stimmt ab über den Bau eines großen Kulturhauses. Im Vorfeld der Abstimmung wird von der Badischen Zeitung, die hier eine monopolartige Stellung hat, eine Umfrage veranstaltet, bei der der scheidende Generalmusikdirektor seine Vorbehalte gegen das Projekt ausspricht. Dafür erhält er einen Brief des Oberbürgermeisters: „Die Stadt Freiburg hat ihre Wünsche jeweils großzügig aufgenommen ... Glauben Sie nicht, daß demgegenüber jetzt Ihr Abgang aus Freiburg ziemlich schäbig aussieht?"

Was geschieht?

Die Badische Zeitung veröffentlicht den Brief, ihr Kulturredakteur Gerhard Jörder formuliert eine äußerst scharfe Glosse an die Adresse des $\mathrm{OB}$, dem man sonst wohlgesonnen ist:

Wer solche Briefe schreibt, zeigt, daß er nichts gelernt hat: ... Wenn die KTS (Kultur- und Tagungsstätte) gebaut wird, dann soll sie gebaut 
werden, weil, nach fairer Abwägung aller Chancen und Risiken, in der Tat mehr für als gegen dieses Unternehmen sprechen mag - nicht aber, weil es sich hier um das Lieblingsprojekt eines OB handelt, der jeden Widerspruch in der Sache offenbar als Majestätsbeleidigung aufzufassen gewillt ist (Badische Zeitung vom 6.7.1988).

An einer solchen Stelle ist die vierte Gewalt intakt. Das klare, offene, entschiedene Wort eines Journalisten in der kleinen Öffentlichkeit einer Stadt ist in der Lage, dem Übergriff der Macht langfristig Grenzen zu setzen.

Der Begriff des 'Pamphlets' wird in unserer Sprache pejorativ gebraucht - vom Blickwinkel des hier vorgetragenen Versuchs aus nicht ganz zu Recht. Vielleicht ist es besser, von Streitschrift zu sprechen. Die Form der wissenschaftlich fundierten umfänglichen Streitschrift ist seit längerem die wohl wirkungsvollste Gattung moralisch-politischer Reflexion. Sie wirft ein neues Licht auf ein großes, allgemein relevantes Tatsachenfeld, bedeutet einen Ruck in seiner Auffassung, löst eine Diskussion aus: indem sie abgewehrt wird, ergreift sie Besitz von der Öffentlichkeit. Sie ist jetzt nicht mehr wegzudenken; ihre Sichtweise hat sich dem öffentlichen Bewußtsein eingezeichnet, 'eingestochen', wie es in Lamprechts Alexanderlied heißt. Ich denke an Bücher wie 'Dialektik der Aufklärung' (Adorno/Horkheimer 1981), 'Die Unwirtlichkeit unserer Städte' (Mitscherlich 1965), 'Medical Nemesis' (Illich 1976), 'Risikogesellschaft' (Beck 1986).

Seit längerem scheint mir noch eine andere Form, der politische Essay, an Bedeutung zu gewinnen. Bildet sich hier eine neue Keimzelle kritischer Politik? Ich zitiere noch einmal Ulrich Beck, aus dem Spiegel Essay 'Eugenik der Zukunft', da mir dieser Autor auf dem von uns erörterten Gebiet eine treffende Sprache zu finden scheint:

Die Eugenik, die uns droht, hat alle Kennzeichen einer finsteren Verschwörung abgelegt und das Kostüm von Gesundheit, Produktivität, Gewinnverheißung angelegt. Ihren Schwung erhält sie durch die Dramaturgie der Gefahren, auf die sie antworten soll (Aids, ökologische Zerstörungen) und die Durchsetzungsmacht von MilliardenInvestitionen. Sie betritt als 'kommerzielle Eugenik' die Bühne der Weltgeschichte (Nr. 47, 1988).

"Ein stummer Gedanke ist ein todter", heißt es bei Jochmann (Jochmann 1837, S. 21). „Wir sind ein gemißhandeltes Volk, weil ein stummes..." (Jochmann 1968, S. 90). Seiner Diagnose zufolge hatte er es in dem Deutschland der 20er Jahre des 19. Jahrhunderts mit einer sprachlosen Öffentlichkeit zu tun.

An dieser Stelle sehe ich eine Parallele. Unsere politische Sprache ist dem, 
was uns alltäglich an Nachrichten erreicht, längst nicht mehr gewachsen, weit hinter dem Geschehen zurückgeblieben. Es verschlägt uns die Sprache. Die Bundesrepublik toleriert seit Jahren den Export von Genozidinstrumenten in Länder, deren erklärtes Ziel die Vernichtung des Staates Israel ist. Die sog. „verbrauchende Forschung" der Gentechnik, ihr Experimentieren mit menschlichen Embryos, läßt die Menschenversuche der KZ-Ärzte in verändertem Licht erscheinen. Was die Industriekonzerne alltäglich anrichten, ist in den Kategorien der Kriminalität längst nicht mehr zu fassen. Sie sind zu idyllisch. Ein Topmanager der Atomindustrie ist so wenig ein Verbrecher, wie ein Atommeiler ein 'Meiler' und ein Entsorgungspark ein 'Park' ist. Welche politische Sprache wäre solchen Sachverhalten gewachsen? Die stärkste Antwort waren in den vergangenen Jahren vielleicht die Ketten der Schweiger und die Graffiti an den Wänden.

\section{V.}

Wenn hier von 'Tendenzen und Perspektiven der Gegenwartssprache' die Rede sein soll, so möchte ich auf eine Lücke hinweisen, genauer, auf ein dünn besetztes Gebiet: auf die Gattungen der Metapolitik und Sozialkunst angesichts der rapiden wissenschaftlich und ökonomisch geleiteten Modernisierung. Diese läuft der politischen Sprache davon. Es scheint mir eine durch große Vorbilder legitimierte Aufgabe der Sprachkritik zu sein, Lücken zu diagnostizieren.

Wir sind von einem historischen Beispiel ausgegangen, von einem wirkungslosen Versuch politischer Sprachkritik und Sprachpolitik aus der Epoche beginnender demokratischer Öffentlichkeit in Deutschland. Jochmann war die Folie für die Frage nach der Gegenwart. Das erste Defizit, das sich zeigte, war eine Geschichte politischer Textgattungen und der Übergangsformen zur Metapolitik: sie wäre ein Indikator des Öffentlichkeitswandels. Die Sprachwissenschaft könnte an ihr mitarbeiten.

Der erste vorläufige Eindruck: Es fehlt die Selbständigkeit des Politischen, analog mangelt es an einer selbständigen politischen Sprache und an wirksamen Formen politisch-moralischer Reflexion.

Als ein Hintergrund erschien der Niedergang der praktischen Philosophie, von dem Hennis und nach ihm andere, Habermas, Apel, Riedel (Habermas 1971; Apel 1972; Riedel 1972), gesprochen haben. Sie ist sehr ähnlich der normativen Sprachwissenschaft und Sprachkritik - von einem naturwissenschaftlich orientierten Wissenschaftsbegriff unterminiert worden. Wir sind dann einige Beispiele politischer Textgattungen und Sprache durchgegangen, sporadisch, fragmentarisch: Portrait, Ge- 
denkrede, Polemik und Kontroverse/Glosse, Streitschrift und Essay.

Der zweite Eindruck, einerseits: Wir finden Beispiele hervorragender politischer Sprache. Es wäre eine mögliche Aufgabe der Sprachkritik, hier zu sichten, hervorzuheben, vielleicht einmal eine Sammlung vorzüglicher Beispiele politischer Rede vorzulegen, vor allem Kriterien der Unterscheidung guter und schlechter politischer Rede zu erarbeiten. Es wäre zudem ein lohnendes Feld der textlinguistischen Arbeit, den jeweiligen Regelkodex der Gattungen von Politik und Metapolitik zu beschreiben - (der beste Zugang zu den Gattungsregeln ist immer das Fallbeispiel einer Regelverletzung) - und die typischen, für die jeweilige Gattung charakteristischen sprachlichen Formen zu analysieren.

Der zweite Eindruck, andrerseits: Es bleibt bei der Vermutung einer Lücke, eines dünn besetzten Gebiets, ja, in mancher Hinsicht einer Sprachlosigkeit.

Dieser Eindruck entsteht offenbar vor allem dadurch, daß es zwar an Gattungen und Formen politischer und metapolitischer Kritik vielleicht nicht mangelt, daß aber diese vom Schweigen bis zur beredten Streitschrift reichenden Ansätze in einer dezentralisierten, diffusen Öffentlichkeit nicht durch das Brennglas einer im öffentlichen Bewußtsein institutionalisierten Kritik gesammelt und fokussiert werden. Im Gegenteil: die Öffentlichkeit ist von der leisen, sanften Diktatur der die Wissenschaft, Wirtschaft und Politik gleichermaßen durchgeisternden Amöben- oder Plastikwörter eingelullt. Alles verpufft, läuft ins Leere, stößt auf Watte, wird zum bloßen Ventil. Was wir meinen, könnte nicht treffender gesagt werden als in einem Passus von Günter Blöcker, der an dieser Stelle allerdings 1959 die literarische Kritik in der Bundesrepublik meinte (Blöcker 1962, S. 13):

Paradox ausgedrückt: wir haben Kritiker, aber wir haben keine Kritik.
Das heißt: wir haben Kritiker, aber sie bleiben Einzelne, von denen jeder
das Risiko der kritischen Existenz für sich allein tragen muß. Wir haben
Kritiker, aber wir haben keine Kritik als Institution. Wobei ich unter In-
stitution beileibe keine staatlich konzessionierte Einrichtung verstehe,
sondern Kritik als selbst verständlichen und allseits anerkannten Be-
standteil des öffentlichen Lebens und des öffentlichen Bewußtseins, als
in die Gesellschaft eingebetteten, sie mittragenden und von ihr mit-
getragenen Faktor, als eine aus eigenem Recht handelnde und auf die
Allgemeinheit einwirkende Macht.

Ich habe versucht, den Umriß meines Themas zu zeichnen, fragmentarisch, unzulänglich - noch etwas unbestimmt - auch aus dem Wunsch, mich nicht sofort in den verbiesterten Positivismus einer Textsortenlinguistik zu verlieren. 
Dieser Beitrag über Textgattungen der Metapolitik mündet in drei Fragen:

1. Welche Gattungen existieren gegenwärtig und welches ist ihr jeweiliger Regelkodex?

2. In welcher Qualität werden diese Gattungen erfüllt?

3. Welche von diesen Gattungen sind besonders geeignet, kritische Metapolitik als Institution (im Sinne Blöckers) zu begründen, und auf welchem Wege wäre ein solcher Gedanke zu fördern?

VI.

Ich komme zum Schluß, kehre zum Vergnügen an Sprache und literarischer Form zurück, in zwölf kurzen abschließenden Punkten.

Was wir von der Sprache und literarischen Form Jochmanns gemeint haben, ließe sich vermutlich auf die Gegenwart übertragen:

1. Form stützt das öffentliche Gedächtnis.

2. Sie erhält dem Geschriebenen einen Schein von Mündlichkeit.

3. Sie ist als Gattung eine Ordnungsform, Spielregel des Zugangs zur Realität, Instrument der Erkenntnis und Urteilsfindung.

4. Sie ist ein intellektuelles Vergnügen und daher gesellig.

5. Sie macht Empörung weltmöglich und daher diskutabel.

6. Sie ist eine scharfe, zugespitzte Waffe.

Im Zeitalter der Massenkommunikation kommen noch weitere Vorzüge hinzu, gewinnt Politik als Sprache und literarische Form noch eine andere Qualität. Ich fasse knapp zusammen in sechs weiteren Punkten:

7. Die Form ist distinkt gegenüber dem großen Stimmendurcheinander und ermöglicht Orientierung.

8. Sie ist ein Werk des Einzelnen, ein Erzeugnis "sozialer Einsamkeit", wenn ich einen Ausdruck Thomas Manns aufgreifen darf (Thomas Mann 1968, S. 158), und auch dadurch ein Gegenmittel.

9. Die Schrift ist das Dauerhafteste von den drei Medien. Sie ist nicht gehört und verweht, gesehen und verwischt. Man hebt sie eher auf. Als geschriebene kann die Form am nachhaltigsten das öffentliche Gedächtnis besetzen.

10. Der Schreibende unterwirft sich inzwischen mehr als der Sprecher in Funk und Fernsehen dem Anspruch, die Stereotypie zu vermei- 
den. Er nimmt sich Zeit, der Normierung zu widerstehen. Das Geschriebene lebt und atmet inzwischen oft mehr als das Gesprochene.

11. Die Gattungen und Formen politischer und moralischer Reflexion können verhindern, daß die Informationslandschaft als Landschaft von Tatsachen mißverstanden wird. Da sie einen höheren Realitätsgrad haben, zersetzen sie die naive Vorstellung, Informationen seien Abbildungen von Tatsachen. Ihre erklärte Subjektivität läßt die Obektivität der Informationslandschaft als falschen Schein erkennen.

12. Die Gattungen politischer und moralischer Reflexion können sich eignen, der Politik die Selbständigkeit zurückzugewinnen und sie zu befähigen, sich gegenüber der Allianz von Naturwissenschaft und Ökonomie zu behaupten und deren zerstörender Wirkung Grenzen zu setzen.

\section{Literatur}

Adorno, Theodor W./Horkheimer, Max (1981): Die Dialektik der Aufklärung. Philosophische Fragmente. Frankfurt/M.: Suhrkamp (Th. W. Adorno, Gesammelte Schriften, hrsg. von Rolf Tiedemann, Bd. 3).

Apel, Karl-Otto (1972): Zum Problem einer rationalen Begründung der Ethik im Zeitalter der Wissenschaft. In: Manfred Riedel (Hrsg.): Rehabilitierung der praktischen Philosophie, 2 Bde., Freiburg: Rombach, Bd. 2, S. 13-32.

Bahr, Egon (September 1984): Das Innere des Äußeren. Ein Gespräch mit Egon Bahr. In: Kursbuch 77, S. 97-110.

Beck, Ulrich (1986): Risikogesellschaft. Auf dem Weg in eine andere Moderne. Frankfurt/M.: Subrkamp (Edition Suhrkamp, Bd. 1365, NF 365).

Belke, Horst (1973): Literarische Gebrauchsformen. Düsseldorf: Bertelsmann Universitätsverlag.

Bentele, Günter/Ruoff, Robert (Hrsg.) (1982): Wie objektiv sind unsere Medien? Frankfurt/M.: Fischer Taschenbuch Verlag.

Blöcker, Günter (1962): Literaturkritik. In: Kritik in unserer Zeit. Literatur. Theater. Musik. Bildende Kunst. Göttingen: Vandenhoek und Ruprecht, S. 46-63.

Burger, Harald (1984): Sprache der Massenmedien. Berlin und New York: de Gruyter (Sammlung Göschen, 2225). 
Camen, Rainer (1984): Die Glosse in der deutschen Tagespresse. Zur Analyse 'journalistigen' Raisonnements. Bochum: Brockmeyer (Bochumer Studien zur Publizistik- und Kommunikationswissenschaft, Bd. 40).

Dieckmann, Walther (1981): Politische Sprache, politische Kommunikation: Vorträge, Aufsätze, Entwürfe. Heidelberg: Winter.

Dovifat, Emil/Wilke, Jürgen (1976): Zeitungslehre, 2 Bde. Bd. 1: Theoretische und rechtliche Grundlagen, Nachricht und Meinung, Sprache und Form. Bd. 2: Redaktion, die Sparten Verlag und Vertrieb, Wirtschaft und Technik, Sicherung der öffentlichen Aufgabe. 6., neubearb. Aufl. Berlin und New York: de Gruyter (Sammlung Göschen 2090 und 2091).

Duwe, Freimut (Mithrsg.) (1977): Briefe zur Verteidigung der Republik, hrsg. von Freimut Duwe, Heinrich Böll, Klaus Staeck. Reinbek bei Hamburg: Rowohlt (= Rhororo 4191).

Enzensberger, Hans Magnus (1988): Das Nullmedium oder Warum alle Klagen gegen das Fernsehen gegenstandslos sind. In: Mittelmaß und Wahn. Gesammelte Zerstreuungen. Frankfurt/M.: Subrkamp, S. 89-103.

Habermas, Jürgen (1971): Kap. 1. In: Erkennt nis und Interesse, Frankfurt/M. Suhrkamp, S. 11-87.

Habermas, Jürgen (1974): Strukturwandel der Öffentlichkeit. Untersuchungen zu einer Kategorie der bürgerlichen Gesellschaft. 6. Aufl. Neuwied und Berlin: Luchterhand (= Ungekürzte Sonderausgabe POLITICA, Bd. 4, hrsg. von Wilhelm Hennis und Roman Schnur).

Hennis, Wilhelm (1963): Politik und praktische Philosophie. Eine Studie zur Rekonstruktion der politischen Wissenschaft. Neuwied und Berlin: Luchterhand (= POLITICA. Abhandlungen und Texte zur politischen Wissenschaft. Hrsg. von Wilhelm Hennis und Hans Maier, Bd. 14).

Historikerstreit (1987): Die Dokumentation der Kontroverse um die Einzigartigkeit der nationalsozialistischen Judenvernichtung. 3. Auf. München und Zürich: Piper (= Serie Piper, Bd. 816):

Illich, Ivan (1976): Limits to medicine. Medical nemesis: the expropriation of health. London: Marion Boyars.

Jochmann, Carl Gustav (1836/1837): Reliquien. Aus seinen nachgelassenen Papieren. Gesammelt von Heinrich Zschokke, 2 Bde., Hechingen: Verlag der Ribler'schen Hofbuchhandlung.

Jochmann, Carl Gustav (1968): Über die Sprache. Faksimiledruck nach der Originalausgabe von 1828, mit Schlabrendorfs „Bemerkungen über die 
Sprache" und der Jochmann-Biographie von Julius Eckardt hrsg. von Christian Johannes Wagenknecht. Göttingen: Vandenhoeck und Ruprecht (= Deutsche Neudrucke. Reihe Texte des 19. Jahrhunderts).

Jochmann, Carl Gustav (1983): Politische Sprachkritik. Aphorismen und Glossen. Hrsg. von Uwe Pörksen. Ausgewählt und kommentiert von Uwe Pörksen und Siegfried Hennrich, Hubert Klausmann, Eva Lange, Jürgen Schiewe. Stuttgart: Reclam.

Koszyk, Kurt/Pruys, Karl H. (Hrsg.) (1970): Wörterbuch zur Publizistik. München-Pullach und Berlin: Verlag Dokumentation (Lizenzausgabe mit Genehmigung des Deutschen Taschenbuchverlages).

Kraft, Werner (Hrsg.) (1967): Carl Gustav Jochmann, Die Rückseite der Poesie und andere Schriften. Frankfurt/M.: Insel.

La Roche, Walther von (1987): Einführung in den praktischen Journalismus. Mit genauer Beschreibung aller Ausbildungswege. Deutschland, Österreich, Schweiz. 10., neu bearb. Aufl. München: List.

Literaturmagazin 21 (1988): Nicolas Born zum Gedenken. Heinrich Mann, heute. Hrsg. von Martin Lüdke und Delf Schmidt. Reinbek bei Hamburg: Rowohlt.

Lüger, Hans Helmut (1983): Pressesprache. Tübingen: Niemeyer (Germanistische Arbeitshefte, Bd. 28).

Mann, Thomas (1968): Rede zur Gründung der Sektion für Dichtkunst der preußischen Akademie der Künste. In: Ders.: Politische Reden und Schriften, Bd. 2. Frankfurt/M. und Hamburg: Fischer Bücherei (Moderne Klassiker 117), S. 156-159.

Mitscherlich, Alexander (1965): Die Unwirtlichkeit unserer Städte. Anstiftung zum Unfrieden. Frankfurt/M.: Suhrkamp (Edition Subrkainp 123).

Noelle-Neumann, Elisabeth/Schulz, Winfried (Hrsg.) (1971): Das Fischer Lexikon Publizistik. Frankfurt/M.: Fischer Taschenbuch Verlag.

Ortheil, Hanns-Josef (1986): Die Staatsschauspieler. In: Merkur 40 II, S. 779 792.

Riedel, Manfred (Hrsg.) (1972): Vorwort. In: Ders. (Hrsg.): Rehabilitierung der praktischen Philosophie, Bd. 1, Freiburg: Rombach (Sammlung Rombach NF. Bd. 23), S. 9-12.

Rohner, Ludwig (1966): Der deutsche Essay. Materialien zur Geschichte und Ästhetik einer literarischen Gattung, Neuwied und Berlin. 
Roloff, Eckart Klaus (1982): Journalistische Textgattungen. München: Oldenbourg (Reihe Studientexte für die Kollegstufe).

Schiewe, Jürgen (1989): Sprache und Öffentlichkeit. Carl Gustav Jochmann und die politische Sprachkritik der Spätaufklärung. Berlin: Erich Schmidt (Philologische Studien und Quellen, H. 118).

Schote, Joachim (1988): Die Entstehung und Entwicklung des deutschen Essays im 18. Jahrhundert. Diss. Freiburg i. Br.

Sennett, Richard (1983): Verfall und Ende des öffentlichen Lebens. Die Tyrannei der Intimität. Aus dem Amerikanischen übersetzt von Reinhard Kaiser. Frankfurt/M.: Fischer (Titel der 1977 bei Alfred A. Knopf, New York, erschienenen Originalausgabe: „The Fall of Public Man”).

Straßner, Erich (Hrsg.) (1975): Nachrichten. Entwicklungen - Analysen - Erfahrungen. München: Fink (Kritische Information 31).

Weck, Roger de (1988): Das Buch als Basis für die Macht. Schreibende Politiker beeindrucken die Franzosen seit jeher. - Ein Aufsatz über 'Literatur und Politik in Frankreich' in der 'Zeit' vom 15. April 1988.

Walther Dieckmann, Josef Kopperschmidt und Erich Straßner danke ich sehr für mündliche und briefliche Anregungen nach dem Vortrag. 